\section{A epidemia de AIDS no Brasil e as desigualdades regionais e de oferta de serviço}

\author{
The AIDS epidemic in Brazil and differences \\ according to geographic region and health \\ services supply
}

\author{
1 Faculdade de Medicina, \\ Universidade de São Paulo, \\ São Paulo, Brasil. \\ 2 Instituto de Saúde, \\ Secretaria de Estado da \\ Saúde de São Paulo, São \\ Paulo, Brasil. \\ Correspondência \\ A. Grangeiro \\ Departamento de Medicina, \\ Faculdade de Medicina \\ Universidade de São Paulo. \\ Rua Dr. Arnaldo 455, São \\ Paulo, SP 01246-903, Brasil. \\ ale.grangeiro@gmail.com
}

\begin{abstract}
The aim of this study was to identify different profiles in the AIDS epidemic in Brazil by relating them to the health sector's organization, situations involving increased risk of infection, and the degree of implementation of the response by health services. The Brazilian municipalities (counties) were grouped according to the magnitude of the epidemic and its trends from 2002 and 2006, and were then studied using indicators obtained from secondary databases. $\mathrm{Mu}$ nicipalities with large epidemics (39\%) displayed more situations associated with risk of infection, and those with an upward trend in incidence (11.5\%) showed a lower degree of response. Cities with large epidemics but with downward or stable trends had $68.6 \%$ of all the anonymous testing centers and $75.8 \%$ of the outpatient clinics, and performed $81.4 \%$ of all the HIV antibody tests in the health system. Preventive measures in schools and primary health services showed low coverage rates. Differences were observed between geographic regions. Inequalities in the degree of implementation of the response to HIV may contribute to different profiles in the epidemic around the country.
\end{abstract}

Acquired Immunodeficiency Syndrome; Health Inequalities; Program Development; Risk
Alexandre Grangeiro 1

Maria Mercedes Loureiro Escuder 2 Euclides Ayres de Castilho 1

\section{Introdução}

A Epidemiologia, em especial a latino-americana, tem uma ampla produção que busca compreender o processo saúde e doença a partir de desigualdades, na qual se enfatiza que formas específicas de adoecimento e morte guardam estreita relação com a organização social e com as condições de vida da população. Compreensão que impõe a necessidade de aprofundar o conhecimento das relações existentes entre aspectos sociais, culturais, econômicos e geográficos, e padrões de ocorrência das doenças e seus agravos 1 .

No campo da AIDS, inúmeras iniciativas neste sentido foram conduzidas desde a descrição dos primeiros casos, em um contexto que procurou romper com abordagens epidemiológicas tradicionais, que circunscrevia o risco de infecção às dimensões individuais e dificultava a apreensão de situações que influenciavam no crescimento da epidemia e nas diferentes formas como as populações eram afetadas 2 . Entre as iniciativas, a de maior expressão e que influenciou vários estudos foi o trabalho realizado por Mann et al. 3, que, adotando um referencial teórico utilizado especialmente na área de direitos humanos, estudou a vulnerabilidade de populações e países para o surgimento e desenvolvimento de epidemias de AIDS.

No Brasil, os estudos desenvolvidos nessa perspectiva foram expressivos, abrangendo uma 
diversidade de áreas e populações, e contribuíram, direta ou indiretamente, para criar um relativo consenso sobre a influência de múltiplas dimensões na definição das principais tendências da epidemia de AIDS no país, como as relativas às diferenças entre gêneros e ao uso de droga 4 . Contribuíram também para orientar o desenho e a implementação de práticas de prevenção e de promoção à saúde, visando à mitigação do impacto da epidemia na população brasileira 5 .

Apesar da predominância desse referencial, são restritos os trabalhos brasileiros que investigaram as relações existentes entre a epidemia de AIDS e os contextos municipais, como o realizado por Szwarcwald et al. 6, demonstrando a ocorrência de diferentes padrões epidemiológicos da AIDS segundo as características sociodemográficas e as formas de transmissão do HIV, predominantes em cidades brasileiras.

São também limitados os estudos que analisaram como a organização da resposta brasileira nas áreas de prevenção e atenção à saúde de pessoas com HIV contribuiu para a ocorrência de diferentes tendências da epidemia no Brasil. Reconhece-se, entretanto, a existência de importantes desigualdades regionais no acesso aos serviços para a AIDS e na apropriação dos resultados positivos atribuídos à resposta brasileira ao HIV, como o início oportuno do tratamento por anti-retroviral (ARV) 7, a redução da taxa de mortalidade 8 e o aumento da população que realizou exames diagnósticos da infecção pelo HIV 9 , que, em geral, apresentam piores indicadores nas regiões Norte e Nordeste.

A relativa escassez desses estudos, sem dúvida contribui para limitar a compreensão dos fatores relacionados às diferentes formas de ocorrência da epidemia de AIDS no Brasil e suas relações com os contextos municipais. Diante desse desafio, este estudo analisa a magnitude e a tendência da epidemia em municípios brasileiros, relacionando-as aos indicadores municipais que caracterizam a capacidade de resposta ao HIV nas áreas de prevenção e atenção à saúde, à presença de situações que indicam possíveis situações de risco e a indicadores epidemiológicos, geopolíticos e de desenvolvimento social.

\section{Metodologia}

Para a análise do perfil epidemiológico os municípios brasileiros foram classificados segundo a magnitude e a tendência da epidemia de AIDS entre os anos de 2002-2006, o que permitiu a identificação de quatro agrupamentos: (a) cidades com ocorrências de grande magnitude e tendência de crescimento da taxa anual de incidência; (b) de grande magnitude e tendência de redução ou estabilização; (c) de pequena magnitude e crescimento acelerado, devido a variações da incidência acima da média nacional; e (d) de pequena magnitude que crescem discretamente ou estabilizam/reduzem. Os perfis epidemiológicos foram estudados, posteriormente, segundo indicadores epidemiológicos, geopolíticos, de implantação da resposta à epidemia e a presença de situações associadas ao risco de infecção pelo HIV.

Os dados foram obtidos de fontes secundárias disponíveis na Internet ou fornecidos pelas instituições mantenedoras dos bancos de dados. As informações epidemiológicas, demográficas e de saúde são provenientes do Departamento de Informática do SUS (http://w3.datasus.gov. br/datasus/index.php?area=02, acessado em 19/ Dez/2008); os geopolíticos e renda média são do Instituto Brasileiro de Geografia e Estatística (http://www.ibge.gov.br/home/estatistica/eco nomia/perfilmunic/defaulttab1_perfil.shtm (acessado em 20/Dez/2008); os de resposta municipal ao HIV originam-se do Departamento Nacional de DST, AIDS e Hepatites Virais (http:// www.aids.gov.br, acessado em 09/Fev/2009), do banco de dados Sistema de Controle de Exames Laboratoriais da Rede Nacional de Contagem de Linfócitos CD4+/CD8+e CargaViral, e do banco de dados cedido pelo Ministério da Educação (Censo Escolar 2006); e os de presença de populações específicas no território foram fornecidos pelo Centro Nacional de Populações Tradicionais e Desenvolvimento Sustentável (http://www.ibama.gov. $\mathrm{br} /$ siucweb/listaUcCategoria.php?abrev=RESEX, acessado em 01/Jul/2007), Ministério da Saúde (http://portal.saude.gov.br/portal/saude/profis sional/visualizar_texto.cfm?idtxt+25625, acessado em 13/Jun/2008), Fundação Nacional do Índio (http://www.funai.gov.br/mapas/fr_mapa_fun diario.htm, acessado em 14/Jun/2008), banco de dados do Núcleo de Estudos, Pesquisa e Projetos de Reforma Agrária, Universidade Estadual Paulista, e Ministério da Justiça (http://www.mj.gov. $\mathrm{br} /$ data/Pages / MJD574E9CEITEMIDAB2EF2 D92895476E8516E63C78FC7C4CPTBRIE.htm, acessado em 11/Set/2008).

A magnitude e a tendência da epidemia foram analisadas para todos os municípios brasileiros com casos de AIDS registrados em três ou mais anos no período de 2002-2006. A magnitude foi estabelecida com base no número total de casos ocorridos no período. Foram considerados de grande magnitude os municípios com ocorrências de 20 ou mais casos, e de pequena magnitude os que apresentaram entre 1 e 19 casos.

Utilizou-se regressão linear simples para analisar a tendência da epidemia em cada mu- 
nicípio 10 , por meio da qual se estimou a variação anual das taxas de incidência entre 2002 e 2006, que foram estimadas com o emprego de médias móveis para cada três anos. Esse procedimento foi usado para minimizar eventuais variações decorrentes de notificações irregulares de casos.

A categorização dos municípios segundo a tendência da epidemia foi realizada em três etapas. Na primeira, estimou-se a variação da taxa anual de incidência para os anos de 2002-2006, sendo classificadas como epidemias em crescimento aquelas com taxas positivas (valor de $\mathrm{p}$ $<0,05)$ e como epidemias em redução, as com taxas negativas.

A segunda etapa compreendeu a análise dos municípios com variação não significativa (valor de $\mathrm{p}>0,05)$ na regressão linear. Para esses municípios analisou-se a dispersão das taxas médias de incidência por meio do coeficiente de variação. Aqueles que apresentaram dispersão inferior a $20 \%$ (coeficiente de variação $\leq 20 \%$ ) foram considerados municípios com epidemias estáveis.

Na terceira etapa, os municípios com dispersão das taxas médias de incidência superior a 20\% (coeficiente de variação > 20\%) tiveram a tendência da epidemia analisada para um período de dez anos (1997-2006), empregando-se regressão linear simples 10 e os mesmos procedimentos de categorização utilizados nas etapas anteriores. A análise de dez anos foi realizada como um esforço para contornar possíveis inconsistências no sistema de notificação que, eventualmente, incidiram nas estimativas realizadas para os anos de 2002-2006.

Municípios classificados pela tendência observada no período de 2002-2006 apresentaram, nas regressões, coeficiente de determinação $\left(\mathrm{R}^{2}\right)$ entre 0,69 e 1,0, e no período de 1997-2006 coeficientes de 0,5 a 0,93. Excluíram-se, pelo baixo grau de explicação do fenômeno, municípios com coeficiente inferior a 0,5. A análise da magnitude e tendência da epidemia realiza com base nessa metodologia pode ser consultada em publicação anterior 11.

Os indicadores epidemiológicos utilizados para a análise dos perfis municipais compreenderam o número total de casos ocorridos entre 2002 e 2006, a incidência média anual no mesmo período, calculada pela média do número de casos nos anos de 2002-2006 e a população de 2004, e a variação do número de casos por categoria de transmissão e sexo, determinada pela razão entre o número de casos ocorridos nos períodos de 2002-2006 e 1997-2001.

Na análise geopolítica estudou-se a distribuição espacial dos municípios por região, unidade federada, tamanho populacional e a inserção do município em região metropolitana, interior ou capital.

Para caracterizar situações relacionadas ao risco de infecção pelo HIV analisaram-se quatro dimensões: (a) presença de populações específicas no território, considerando a existência de assentamentos rurais entre 1979 e 2007, reservas indígenas e extrativistas legalizadas (2008), populações remanescentes de quilombolas em 2008 e localização do município em região de fronteira; (b) vulnerabilidade de mulheres, demonstrada pela desigualdade entre gêneros e/ou prática sexual "desprotegida" acima da média nacional. Foram categorizados como vulneráveis os municípios com dois ou mais indicadores, sendo eles: razão entre rendimentos médios de homens e mulheres em 2000, proporção média de nascidos vivos com mães entre 15 e 19 anos de 2005 a 2007 , número médio de internações por aborto para mil mulheres entre 10 e 49 anos de 2005 a 2007 e coeficiente de mortalidade por câncer de colo de útero entre mulheres de 25-59 anos de 2005 a 2007; (c) o uso de drogas como problema de Saúde Pública, excetuando o álcool, foi dimensionado por meio do número médio de internações por este agravo no SUS por 10 mil habitantes entre 10 e 49 anos de 2005 a 2007; e (d) a existência de unidades prisionais no território em 2008.

A resposta municipal ao HIV foi caracterizada pela realização de duas ou mais ações nas áreas de prevenção e de atenção à saúde de pessoas com HIV, sendo elas: (a) número médio acima da média nacional de exames anti-HIV por mil habitantes entre 2005 e 2007; (b) existência de Centros de Testagem e Aconselhamento (CTA) em 2005; (c) número médio acima da média nacional de procedimentos de doenças sexualmente transmissíveis (DST) e AIDS na atenção básica por 100 habitantes entre 2005 e 2007; e (d) pelo menos $75 \%$ das escolas de ensinos fundamental e médio realizando ações de prevenção da AIDS em 2006. Para a atenção à saúde: (a) estrutura para o cuidado de pessoas com HIV, considerando a existência de ambulatórios especializados em 2007; (b) a realização de internação de pacientes entre 2005 e 2007; e (c) a qualidade do seguimento de pacientes, dimensionado pela proporção média acima da média de exames de contagem de células T-CD4+/CD8+ realizada por paciente/ano entre 2003 e 2006. A gestão de programas de AIDS foi dimensionada pela inclusão dos municípios na Política de Incentivo do Ministério da Saúde em 2006, que é responsável pelo repasse de recursos aos municípios para o desenvolvimento de ações descentralizadas de controle da doença.

A associação entre características dos perfis epidemiológicos e dos indicadores foi analisada 
por meio da estatística de qui-quadrado (variáveis categóricas) e, quando significantes com valores de $\mathrm{p}<0,05$, aplicou-se a análise de resíduos padronizados (Zres), assumindo como fora do padrão casos com valores acima de $|1,96| 12$. Para variáveis contínuas empregou-se ANOVA, recorrendo-se à desigualdade de Bonferroni para testar múltiplas diferenças entre médias.

\section{Resultados}

\section{Magnitude e tendência da epidemia}

Dois mil trezentos e trinta e dois municípios brasileiros registraram casos de AIDS em, no mínimo, três anos no período de 2002-2006, sendo que para 1.910, que constituirão a população deste estudo, foi possível estabelecer a tendência da epidemia. Esses municípios correspondiam a 96\% (178.766) do total de casos (186.283) ocorridos no Brasil entre 2002-2006; a uma incidência média anual de 24,9 casos por 100 mil habitantes; e a 45,6\% dos 4.190 municípios com casos registrados nos anos analisados.

Os 422 municípios excluídos do estudo pela impossibilidade de interpretação da tendência da epidemia segundo os critérios estatísticos definidos, concentraram-se em cidades de pequeno porte $(94,8 \%)$, com até 50 mil habitantes (89,8\%) e apresentaram ocorrências de pequena magnitude (menos de 19 casos no período).

Do conjunto de 1.910 municípios (Figura 1), 744 apresentaram epidemias de grande magnitude, sendo que em 219 (11,5\% do total) a tendência observada foi de crescimento, com uma variação média anual da taxa de incidência de 2,6 casos por 100 mil habitantes. Nos outros 525 municípios (27,5\% do total) a tendência foi de redução/estabilização, com variação anual de -1,4 caso por 100 mil habitantes.

O conjunto com epidemias de pequena magnitude reuniu 1.166 municípios, dos quais 238 (12,5\% do total) tiveram um crescimento acelerado, apresentando uma variação anual de 4,7 casos de AIDS por 100 mil habitantes; aqueles com tendência de crescimento discreto ou de estabilização/redução representaram 928 municípios (48,6\%, do total), com uma variação média anual de $-0,4$ caso por 100 mil habitantes.

Comparando os períodos de 1999/2001 e 2002/2006, os municípios com ocorrências de grande magnitude e em crescimento apresentaram incremento acima da média nacional nas principais categorias de transmissão (Tabela 1). Entre os heterossexuais o crescimento foi de 2,8, entre homo/bissexuais 1,6 e entre os usuários de drogas injetáveis 1,2 - o único grupo de cidades que apresentou tendência de crescimento nesta última categoria de transmissão. Observouse nesses municípios, também, a maior taxa de crescimento entre as mulheres (3,8 vezes), que foi $52 \%$ acima da média nacional.

Como conseqüência, a participação proporcional desse grupo no total de casos aumentou em $37,5 \%$ no período analisado, correspondendo a 12,7\% (22.742) das ocorrências registradas entre 2002 e 2006. A taxa de incidência média anual, a segunda em importância, foi de 19,8 casos por 100 mil habitantes (Tabela 2).

Por sua vez, a redução/estabilização de novos casos em epidemias de grande magnitude ocorreu mantendo elevadas taxas de incidência, 25,6 casos por 100 mil habitantes, a maior entre os grupos analisados, e a redução/estabilização foi restrita às categorias de transmissão UDI $(0,9)$, receptores de sangue e derivados $(0,5)$ e vertical $(0,9)$. Entre homossexuais, a epidemia cresceu 1,3 vez, entre heterossexuais 1,6 e entre as mulheres 2,0. Esses municípios reúnem, ainda, 81,7\% (146.064) das ocorrências registradas entre 2002 e 2006 (Tabela 2).

Nos municípios com epidemias de pequena magnitude, a variação no número de casos esteve fortemente relacionada à categoria de transmissão heterossexual, que foi a única que apresentou tendência de crescimento no período analisado, especialmente em cidades que cresceram acima da média nacional ( 2,8 vezes). Nas demais categorias de transmissão, a redução/estabilização foi, em sua maioria, mais intensa do que as observadas nacionalmente ou nas cidades com grande magnitude (Tabela 1).

No total, os municípios com pequena magnitude representaram $5,6 \%$ do total de casos registrados entre 2002 e 2006, com uma incidência que variou entre 16 casos por 100 mil habitantes nas cidades que cresceram acima da média nacional e 10,1 casos por 100 mil habitantes nas cidades que cresceram discretamente/reduziram. Ressalta-se que entre os períodos de 1997-2001 e 2002-2006 os municípios que cresceram acima da média apresentaram um aumento no número total de casos na ordem de 1,6 vez. Esse valor é $24,1 \%$ superior ao observado para as cidades com grande magnitude (Tabela 2).

Os municípios com ocorrências de grande magnitude apresentaram características demográficas compatíveis com os principais centros urbanos do país (Tabela 2), sendo que aqueles com tendência de redução/estabilização possuíam maior porte populacional, com média de 194.191 habitantes, reuniam $67,9 \%$ da população dos municípios analisados e concentraram-se nos estados do Rio de Janeiro $(50 \%$, Zres $=4,4)$, São Paulo (48,7\%, Zres = 9,9), Rio Grande do Sul 


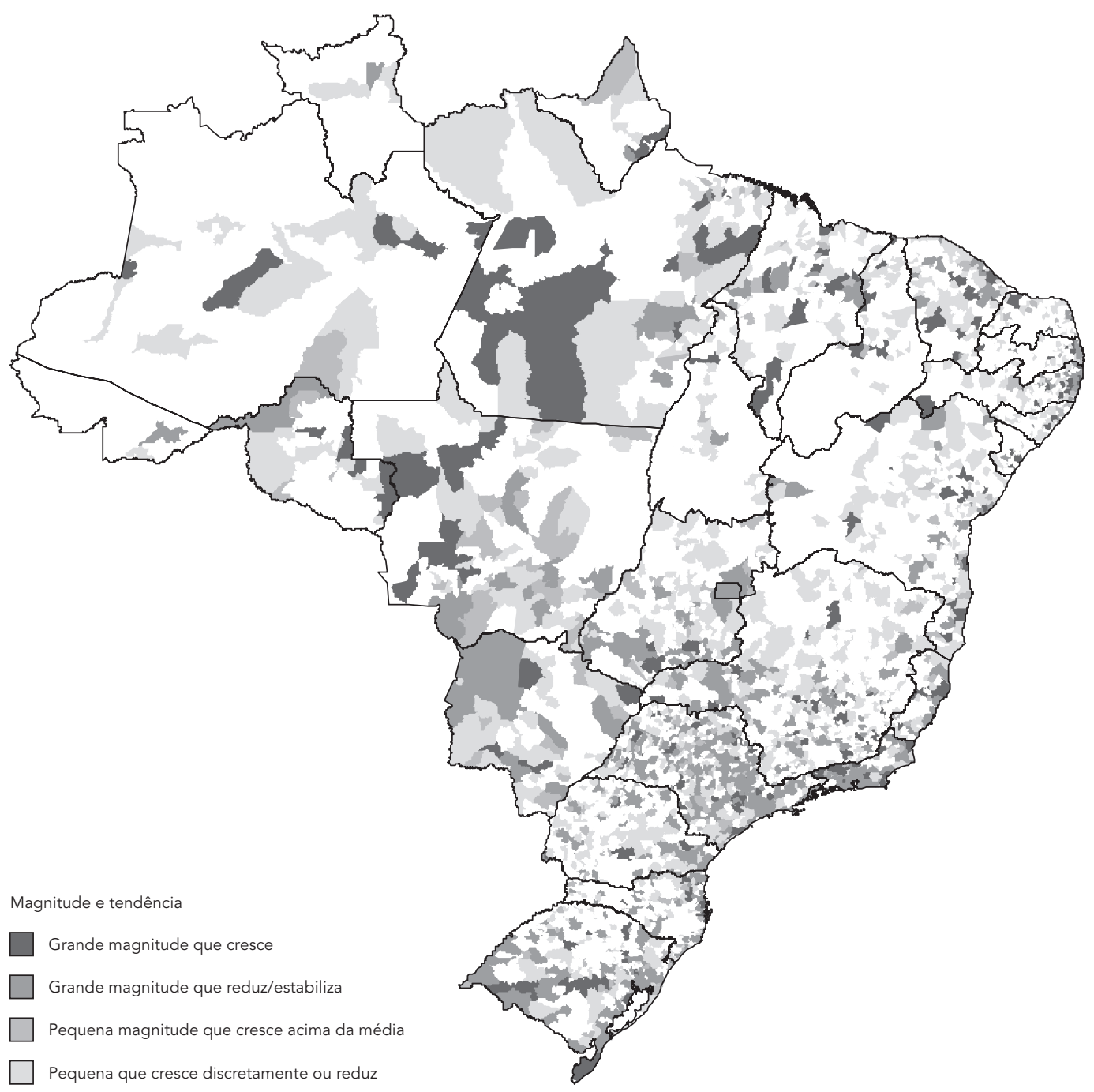

$(39,2 \%$, Zres $=3,3)$ e Santa Catarina $(36,8 \%$, Zres $=2,1$ ). Dentre essas cidades estão vinte capitais brasileiras, $60,8 \%$ dos municípios inseridos em regiões metropolitanas e $80,1 \%$ dos com mais de 100 mil habitantes.

Por outro lado, o grupo com tendência de crescimento concentrou-se nos municípios de médio porte populacional, entre 50-199 mil habitantes (média de 107.849), nas regiões Norte (Zres $=2,6$ ) e Nordeste (Zres $=3,4$ ) e incluiu 50 cidades com mais de 100 mil habitantes e 7 capitais (Tabela 2).

A ocorrência de menor magnitude foi mais prevalente entre cidades de pequeno porte, sen- do que 79,8\% (Zres = 14,8) das com epidemias que cresceram acima da média nacional possuíam até 19 mil habitantes (média de 13.781 habitantes) e localizavam-se no interior dos estados (95\%, Zres $=4,3)$, especialmente no Mato Grosso $(27,9 \%$, Zres $=3,7)$ e Rio Grande do Norte $(25,8 \%$, Zres $=2,3$ ).

É importante frisar que, embora haja a predominância de epidemias grandes em cidades de maior porte populacional e pequenas em cidades com menor número de habitantes, epidemias grandes e pequenas foram observadas em locais de diferentes tamanhos populacionais (Tabela 2). Assim, três cidades entre 100 e 199 
Tabela 1

Incremento do número de novos casos de AIDS por categoria de transmissão do HIV, calculado por meio da razão entre os casos registrados de $1997-2001$ e de 2002-2006. Municípios brasileiros com casos de AIDS registrados em pelo menos três anos no período de 2002-2006.

\begin{tabular}{|c|c|c|c|c|c|}
\hline \multirow[t]{3}{*}{ Categoria de transmissão } & \multicolumn{4}{|c|}{ Incremento dos casos por perfil epidemiológico } & \multirow{3}{*}{$\begin{array}{l}\text { Total } \\
\text { Razãc }\end{array}$} \\
\hline & $\begin{array}{c}\text { Grandes que } \\
\text { crescem }\end{array}$ & $\begin{array}{c}\text { Grandes que } \\
\text { reduzem/ } \\
\text { estabilizam }\end{array}$ & $\begin{array}{l}\text { Pequenas que } \\
\text { crescem } \\
\text { aceleradamente }\end{array}$ & $\begin{array}{l}\text { Pequenas que } \\
\text { crescem } \\
\text { discretamente/ } \\
\text { reduzem }\end{array}$ & \\
\hline & Razão & Razão & Razão & Razão & \\
\hline Heterossexual & 2,8 & 1,6 & 2,8 & 2,3 & 2,2 \\
\hline Homo/Bissexual & 1,6 & 1,3 & 1,1 & 1,0 & 1,2 \\
\hline Uso de drogas injetáveis & 1,2 & 0,9 & 0,7 & 0,7 & 0,9 \\
\hline Recepção de sangue/hemoderivados & 0,5 & 0,5 & 0,3 & 0,4 & 0,5 \\
\hline Transmissão vertical & 1,1 & 0,9 & 0,8 & 0,7 & 0,9 \\
\hline Mulheres & 3,8 & 2,0 & 2,9 & 2,5 & 2,5 \\
\hline
\end{tabular}

Tabela 2

Indicadores epidemiológicos e geopolíticos por perfil epidemiológico. Municípios brasileiros com casos de AIDS registrados em pelo menos três anos no período de 2002-2006.

\begin{tabular}{|c|c|c|c|c|c|c|c|c|c|c|c|c|c|c|}
\hline \multirow[t]{3}{*}{ Indicadores } & \multicolumn{12}{|c|}{ Magnitude e tendência da epidemia municipal } & \\
\hline & \multicolumn{3}{|c|}{$\begin{array}{l}\text { Grandes que } \\
\text { crescem }\end{array}$} & \multicolumn{3}{|c|}{$\begin{array}{c}\text { Grandes que } \\
\text { reduzem/estabilizam }\end{array}$} & \multicolumn{3}{|c|}{$\begin{array}{c}\text { Pequenas } \\
\text { que crescem } \\
\text { aceleradamente }\end{array}$} & \multicolumn{3}{|c|}{$\begin{array}{l}\text { Pequenas que } \\
\text { crescem discreta- } \\
\text { mente/ reduzem }\end{array}$} & \multirow[b]{2}{*}{$\mathbf{n}$} & \multirow[b]{2}{*}{$\%$} \\
\hline & n & $\%$ & Zres & $\mathrm{n}$ & $\%$ & Zres & n & $\%$ & Zres & $\mathrm{n}$ & $\%$ & Zres & & \\
\hline \multicolumn{15}{|l|}{ Epidemiológicos } \\
\hline População 2006 & 23.619 .007 & 15,7 & - & 101.950 .463 & 67,9 & - & 3.279 .861 & 2,2 & - & 21.335 .906 & 14,2 & - & 150.185 .237 & 100,0 \\
\hline $\begin{array}{l}\text { Casos de AIDS } \\
\text { entre } 1997 \text { e } \\
2001\end{array}$ & 13.026 & 9,3 & - & 12.2970 & 87,3 & - & 809 & 0,6 & - & 3.998 & 2,8 & - & 140.803 & 100,0 \\
\hline $\begin{array}{l}\text { Casos de AIDS } \\
\text { entre } 2002 \text { e } \\
2006\end{array}$ & 22.742 & 12,7 & - & 146.064 & 81,7 & - & 2.092 & 1,2 & - & 7.868 & 4,4 & - & 178.766 & 100,0 \\
\hline $\begin{array}{l}\text { Incidência de } \\
\text { AIDS média } \\
\text { por } 100 \text { mil } \\
\text { habitantes (2006) }\end{array}$ & 19,8 & - & - & 25,6 & - & - & 16,0 & - & - & 10,1 & - & - & 16,1 & - \\
\hline \multicolumn{15}{|l|}{ Geopolíticos } \\
\hline $\begin{array}{l}\text { Número de } \\
\text { municípios }\end{array}$ & 219 & 11,5 & - & 525 & 27,5 & - & 238 & 12,5 & - & 928 & 22,1 & - & 1.910 & 100,0 \\
\hline $\begin{array}{l}\text { Número de } \\
\text { capitais }\end{array}$ & 7 & 25,9 & 2,4 & 20 & 74,1 & 5,5 & - & - & $-2,0$ & - & - & $-5,1$ & 27 & 100,0 \\
\hline $\begin{array}{l}\text { Municípios } \\
\text { em Região } \\
\text { Metropolitana }\end{array}$ & 32 & 13,1 & 0,8 & 149 & 60,8 & 12,5 & 12 & 4,9 & $-3,8$ & 52 & 21,2 & $-9,2$ & 245 & 100,0 \\
\hline $\begin{array}{l}\text { Municípios no } \\
\text { interior }\end{array}$ & 180 & 11,0 & $-1,6$ & 356 & 21,7 & $-13,8$ & 226 & 13,8 & 4,3 & 876 & 53,5 & 10,5 & 1.638 & 100,0 \\
\hline
\end{tabular}


Tabela 2 (continuação)

\begin{tabular}{|c|c|c|c|c|c|c|c|c|c|c|c|c|c|c|}
\hline \multirow[t]{3}{*}{ Indicadores } & \multicolumn{12}{|c|}{ Magnitude e tendência da epidemia municipal } & \multicolumn{2}{|c|}{ Total } \\
\hline & \multicolumn{3}{|c|}{$\begin{array}{c}\text { Grandes que } \\
\text { crescem }\end{array}$} & \multicolumn{3}{|c|}{$\begin{array}{c}\text { Grandes que } \\
\text { reduzem/estabilizam }\end{array}$} & \multicolumn{3}{|c|}{$\begin{array}{c}\text { Pequenas } \\
\text { que crescem } \\
\text { aceleradamente }\end{array}$} & \multicolumn{3}{|c|}{$\begin{array}{l}\text { Pequenas que } \\
\text { crescem discreta- } \\
\text { mente/ reduzem }\end{array}$} & \multirow[b]{2}{*}{$\mathrm{n}$} & \multirow[b]{2}{*}{$\%$} \\
\hline & $n$ & $\%$ & Zres & $\mathrm{n}$ & $\%$ & Zres & $\mathrm{n}$ & $\%$ & Zres & $n$ & $\%$ & Zres & & \\
\hline \multicolumn{15}{|l|}{ Número de } \\
\hline \multicolumn{15}{|l|}{ habitantes (mil) } \\
\hline Mais de 500 & 9 & 25,0 & 2,6 & 27 & 75,0 & 6,4 & - & - & $-2,3$ & - & - & $-5,9$ & 36 & 100,0 \\
\hline $200-499$ & 11 & 11,7 & 0,1 & 83 & 88,3 & 13,5 & - & - & $-3,8$ & - & - & $-9,7$ & 94 & 100,0 \\
\hline 100-199 & 30 & 21,9 & 4,0 & 104 & 75,9 & 13,2 & - & - & $-4,6$ & 3 & 2,2 & 11,3 & 137 & 100,0 \\
\hline $50-99$ & 72 & 25,6 & 8,1 & 154 & 54,8 & 11,1 & - & - & $-6,8$ & 55 & 19,6 & $-10,5$ & 281 & 100,0 \\
\hline $20-49$ & 85 & 12,8 & 1,3 & 133 & 20,0 & $-5,3$ & 48 & 7,2 & $-5,1$ & 398 & 59,9 & 7,2 & 664 & 100,0 \\
\hline Até 19 & 12 & 1,7 & 10,1 & 24 & 3,4 & $-17,9$ & 190 & 27,2 & $-14,8$ & 472 & 67,6 & 12,6 & 698 & 100,0 \\
\hline População média & 107.849 & - & - & 194.191 & - & - & 13.781 & - & - & 22951 & - & - & 78.631 & - \\
\hline \multicolumn{15}{|l|}{ Região do país } \\
\hline Norte & 23 & 18,5 & 2,6 & 11 & 8,9 & $-4,8$ & 14 & 11,3 & $-0,4$ & 76 & 61,3 & 2,9 & 124 & 100,0 \\
\hline Centro-oeste & 14 & 7,3 & $-1,9$ & 39 & 20,3 & $-2,3$ & 39 & 20,3 & 3,5 & 100 & 52,1 & 1,0 & 192 & 100,0 \\
\hline Nordeste & 79 & 15,6 & 3,4 & 61 & 12,1 & $-9,0$ & 55 & 10,9 & $-1,2$ & 310 & 61,4 & 6,7 & 505 & 100,0 \\
\hline Sudeste & 71 & 9,9 & $-1,7$ & 277 & 38,5 & 8,4 & 79 & 11,0 & $-1,5$ & 292 & 40,6 & $-5,4$ & 719 & 100,0 \\
\hline Sul & 32 & 8,6 & $-1,9$ & 137 & 37,0 & 4,6 & 51 & 13,8 & 0,9 & 150 & 40,5 & $-3,4$ & 370 & 100,0 \\
\hline
\end{tabular}

mil habitantes possuíam epidemias pequenas com crescimento discreto/redução, e 12 municípios com menos de 19 mil habitantes tiveram epidemias grandes em crescimento, sendo que somente em quatro observou-se a presença de unidades prisionais.

\section{O risco e a resposta à AIDS segundo perfis epidemiológicos}

As cidades com epidemias de grande magnitude estiveram mais fortemente associadas aos contextos que caracterizam o risco, apresentando as maiores proporções de cidades que possuíam: (1) dois ou mais indicadores que sugeriam diferenças entre gêneros e/ou práticas sexuais desprotegidas entre mulheres; (2) populações específicas em seu território, como assentamentos rurais, reservas indígenas e quilombos; (3) unidades prisionais implantadas; e (4) as maiores taxas médias de internação por uso de drogas, excetuando álcool (Tabela 3).

São esses municípios, também, que apresentaram o maior grau de implantação da resposta ao HIV (Tabela 4), que esteve, contudo, mais fortemente concentrada nas cidades com epidemias de grande magnitude com tendência de redução ou estabilização. Esse grupo foi o que mais concentrou ações de diagnóstico (56,8\%; Zres = 19,1), de atenção à saúde de pessoas com $\operatorname{HIV}(78,9 \%$; Zres $=19,7$ ) e de organização de programas para o enfretamento da doença $(66,5 \%$; Zres $=26,5)$. Reuniu 75,8\% (Zres = 21,5) dos municípios com ambulatórios especializados para AIDS, 68,6\% (Zres $=16,4)$ dos CTA, 81,4\% dos testes anti-HIV realizados no SUS entre 2003 e 2007 e 75,2\% (Zres $=26,5)$ das cidades que receberam recursos do Ministério da Saúde para o desenvolvimento de ações programáticas de DST e AIDS. Adicionalmente, o número médio de exames de contagem de células T-CD4+/CD8+ por paciente/ano ( 1,8 exame) nesses municípios foi maior do que os demais grupos analisados.

Excluindo os municípios com epidemias de grande magnitude que reduziram/estabilizaram, a resposta ao HIV tendeu a ser restrita. Por exemplo, em 74,9\% dos municípios de grande magnitude que cresceram não existem ambulatórios específicos para AIDS, 64,4\% não receberam recursos do Ministério da Saúde para o controle da AIDS, e somente $15,7 \%$ dos exames anti-HIV realizados no SUS foram provenientes desses municípios.

No grupo de pequenas epidemias, essas características foram mais acentuadas, ressaltando-se que, somente $2,1 \%$ (Zres $=-21,9$ ) possuíam ambulatórios especializados e 53,3\% (Zres $=18,6$ ) não registraram internação de pessoas com HIV no período analisado. A exceção refere-se ao número médio de exames de contagem de células T-CD4+/CD8+ por paciente/ano (1,7 exame), que foi similar ao das cidades com epi- 
Tabela 3

Situações que favorecem o risco de infecção pelo HIV, presença de populações específicas e estabelecimentos prisionais no território segundo perfil epidemiológico. Municípios brasileiros com casos de AIDS registrados em pelo menos três anos no período de $2002-2006$.

\begin{tabular}{|c|c|c|c|c|c|c|c|c|c|}
\hline \multirow[t]{3}{*}{ Indicadores } & \multicolumn{8}{|c|}{ Perfil epidemiológico } & \multirow{3}{*}{$\begin{array}{c}\text { Total } \\
\\
\%\end{array}$} \\
\hline & \multicolumn{2}{|c|}{$\begin{array}{l}\text { Grandes que } \\
\text { crescem }\end{array}$} & \multicolumn{2}{|c|}{$\begin{array}{l}\text { Grandes que } \\
\text { reduzem/ } \\
\text { estabilizam }\end{array}$} & \multicolumn{2}{|c|}{$\begin{array}{l}\text { Pequenas } \\
\text { que crescem } \\
\text { aceleradamente }\end{array}$} & \multicolumn{2}{|c|}{$\begin{array}{c}\text { Pequenas } \\
\text { que crescem } \\
\text { discretamente/ } \\
\text { reduzem }\end{array}$} & \\
\hline & $\%$ & Zres & $\%$ & Zres & $\%$ & Zres & $\%$ & Zres & \\
\hline \multicolumn{10}{|l|}{ Relações desprotegidas e diferença entre gêneros } \\
\hline $\begin{array}{l}\text { \% de municípios com } 2 \text { ou mais indicadores de relações } \\
\text { sexuais desprotegidas e diferenças entre gêneros acima } \\
\text { da média nacional }\end{array}$ & 69,2 & 3,7 & 62,9 & 2,5 & 52,8 & $-2,4$ & 55,3 & $-2,8$ & 58,2 \\
\hline $\begin{array}{l}\text { \% com razão entre rendimentos médios de homens e } \\
\text { mulheres acima da média nacional ( } 1,5 \mathrm{vez})\end{array}$ & 47,2 & $-0,8$ & 62,8 & 7,0 & 47,0 & $-1,1$ & 44,6 & $-4,6$ & 49,6 \\
\hline $\begin{array}{l}\text { \% com taxa de internações por aborto acima da média } \\
\text { nacional }(8,7 \text { por } 10 \text { mil mulheres de } 10-49 \text { anos })\end{array}$ & 62,0 & 3,9 & 62,4 & 6,3 & 40,4 & $-4,3$ & 45,6 & $-4,6$ & 50,5 \\
\hline $\begin{array}{l}\text { \% com coeficiente de mortalidade por câncer de colo de } \\
\text { útero acima da média nacional }(0,6 \text { por } 10 \text { mil mulheres } \\
\text { de } 25-59 \text { anos) }\end{array}$ & 44,8 & 6,3 & 39,7 & 6,9 & 20,1 & $-3,8$ & 21,2 & $-7,0$ & 28,0 \\
\hline $\begin{array}{l}\text { \% de nascidos vivos com mães entre } 15-19 \text { anos acima da } \\
\text { média }(22,9 \%)\end{array}$ & 41,2 & $-1,1$ & 19,0 & $-13,5$ & 57,5 & 5,7 & 52,9 & 7,9 & 44,3 \\
\hline \multicolumn{10}{|l|}{ Presença de populações específicas (\%) } \\
\hline Com pelo menos uma população específica & 52,8 & 2,2 & 40,6 & $-2,9$ & 48,8 & 1,1 & 46,4 & 0,2 & 46,1 \\
\hline Reserva extrativista legalizada & 1,6 & 0,5 & 0,9 & $-0,7$ & 1,3 & 0,2 & 1,3 & 0,2 & 1,2 \\
\hline Reserva indígena legalizada & 12,0 & 2,9 & 7,3 & $-0,2$ & 6,6 & $-0,7$ & 6,9 & $-1,1$ & 7,5 \\
\hline Remanescentes de comunidades quilombolas & 11,6 & 1,3 & 10,2 & 0,8 & 7,9 & $-1,1$ & 9,0 & $-0,7$ & 9,4 \\
\hline Região de fronteira & 1,6 & $-0,7$ & 2,0 & $-0,2$ & 2,1 & $-0,1$ & 2,4 & 0,7 & 2,2 \\
\hline Assentamento rural & 46,8 & 2,5 & 31,8 & $-4,1$ & 42,0 & 1,1 & 40,6 & 1,1 & 39,4 \\
\hline$\%$ com unidades prisionais implantadas & 41,6 & 7,0 & 38,0 & 8,9 & 13,2 & $-5,3$ & 16,4 & $-8,0$ & 23,8 \\
\hline Uso de drogas como problema de saúde pública & $\mathrm{n}$ & IC95\% & $\mathrm{n}$ & IC95\% & $n$ & IC95\% & $n$ & IC95\% & $n$ \\
\hline $\begin{array}{l}\text { Taxa média de internação por uso de drogas, excetuando } \\
\text { álcool, por } 10 \text { mil habitantes entre } 10-49 \text { anos }\end{array}$ & 5,2 & $4,2-6,1$ & 8,5 & $7,7-9,3$ & 3,8 & $3,2-4,5$ & 3,7 & $3,4-4,1$ & 5,0 \\
\hline
\end{tabular}

IC95\%: intervalo de 95\% de confiança.

demias de grande magnitude em crescimento (Tabela 4).

Nota-se que, para o conjunto dos grupos analisados, a maior restrição da resposta ao HIV foi observada na área da prevenção, onde nenhuma das ações estudadas abrangeu mais de $40 \%$ dos municípios incluídos na análise. As grandes epidemias, especialmente as que reduziram, foram as que registraram as menores proporções de municípios que realizaram ações de prevenção em ao menos $75 \%$ das escolas (18,7\%; Zres $=-6,1)$ ou que disponibilizaram, em patamares superiores à média nacional, consultas de DST e AIDS na atenção básica $(8,2 \%$; Zres = $-6,2)$. Por sua vez, as pequenas epidemias foram as que menos ofertaram duas ou mais ações de prevenção $(88,8 \%$; Zres = 11,1), observando-se maior restrição à oferta de exames anti-HIV ou ter CTA implantado no município (91,8\%; Zres = 91,8) (Tabela 4).

Ressalta-se que os grupos com epidemias de pequena magnitude apresentaram, comparativamente, uma menor proporção de municípios com indicadores de risco para infecção pelo HIV. Entretanto, é importante observar que 55,2\% apresentaram situações de desigualdade entre gêneros e/ou mulheres com relações sexuais desprotegidas, e 48,2\% possuíam em seu território populações associadas à vulnerabilidade do HIV (Tabela 3).

\section{Discussão}

Estudos ecológicos constituem importantes instrumentos para a definição de políticas públicas, 
Tabela 4

Indicadores de implantação da resposta ao HIV nas áreas de atenção às pessoas com HIV, prevenção das DST/AIDS e ações programáticas por perfil epidemiológico. Municípios com casos de AIDS registrados em pelo menos três anos no período de 2002-2006.

\begin{tabular}{|c|c|c|c|c|c|c|c|c|c|}
\hline \multirow[t]{3}{*}{ Indicadores } & \multicolumn{8}{|c|}{ Perfil epidemiológico } & \multirow{3}{*}{$\begin{array}{c}\text { Total } \\
\text { Valor de }\end{array}$} \\
\hline & \multicolumn{2}{|c|}{ Grandes que crescem } & \multicolumn{2}{|c|}{$\begin{array}{c}\text { Grandes que } \\
\text { reduzem/estabilizam }\end{array}$} & \multicolumn{2}{|c|}{$\begin{array}{l}\text { Pequenas que crescem } \\
\text { aceleradamente }\end{array}$} & \multicolumn{2}{|c|}{$\begin{array}{l}\text { Pequenas } \\
\text { que crescem } \\
\text { discretamente/ } \\
\text { reduzem }\end{array}$} & \\
\hline & Valor de $p$ & Zres & Valor de $p$ & Zres & Valor de $p$ & Zres & Valor de $\mathrm{p}$ & Zres & \\
\hline \multicolumn{10}{|l|}{ Prevenção das DST e AIDS } \\
\hline $\begin{array}{l}\text { \% que realizam } 2 \text { ou mais } \\
\text { atividades de prevenção }\end{array}$ & 29,7 & 4,2 & 32,8 & 9,2 & 16,4 & $-1,2$ & 9,9 & $-10,1$ & 19,3 \\
\hline$\%$ com CTA & 26,5 & 5,5 & 35,4 & 16,4 & 1,7 & $-5,9$ & 2,5 & $-14,3$ & 14,2 \\
\hline $\begin{array}{l}\text { Média de exames HIV realizados } \\
\text { por } 1.000 \text { habitantes }\end{array}$ & 19,4 * & - & 28,0 ** & - & 15,1 & - & 10,5 * & - & 20,0 \\
\hline $\begin{array}{l}\text { \% que realizam diagnóstico do } \\
\text { HIV acima da média nacional }(17,1 \\
\text { testes por } 1 \text { mil habitantes) }\end{array}$ & 33,8 & 5,2 & 45,9 & 17,0 & 7,6 & $-5,3$ & 6,3 & $-15,0$ & 20,5 \\
\hline $\begin{array}{l}\text { \% com atividades de prevenção } \\
\text { em pelo menos } 75 \% \text { escolas }\end{array}$ & 26,0 & $-1,0$ & 18,7 & $-6,1$ & 36,1 & 2,6 & 33,6 & 4,4 & 29,0 \\
\hline \multicolumn{10}{|l|}{$\begin{array}{l}\text { AIDS na atenção básica por } 100 \\
\text { habitantes }\end{array}$} \\
\hline $\begin{array}{l}\text { \% de consultas de DST e AIDS na } \\
\text { atenção básica acima da média } \\
\text { nacional (5,5 por } 100 \text { habitantes) }\end{array}$ & 13,7 & $-1,3$ & 8,2 & $-6,2$ & 31,9 & 6,6 & 18,6 & 2,0 & 16,9 \\
\hline $\begin{array}{l}\text { \% que realizam atividades de } \\
\text { prevenção em pelo menos } 75 \% \\
\text { das escolas ou possui número de } \\
\text { consultas de DST/AIDS na atenção } \\
\text { básica acima da média nacional }\end{array}$ & 35,6 & $-1,5$ & 25,3 & $-8,2$ & 56,7 & 5,5 & 45,8 & 4,7 & 40,4 \\
\hline $\begin{array}{l}\text { \% que possui CTA ou realiza } \\
\text { diagnóstico acima da média } \\
\text { nacional }\end{array}$ & 44,7 & 6,8 & 56,8 & 19,1 & 8,4 & $-6,5$ & 8,2 & $-17,1$ & 25,8 \\
\hline \multicolumn{10}{|l|}{ Atenção à saúde de pessoas com HIV } \\
\hline $\begin{array}{l}\text { \% que realizam } 2 \text { ou mais ações } \\
\text { de atenção à saúde }\end{array}$ & 56,2 & 4,3 & 78,9 & 19,7 & 22,7 & $-6,7$ & 24,2 & $-15,9$ & 42,7 \\
\hline $\begin{array}{l}\text { \% de municípios com } \\
\text { ambulatórios para AIDS }\end{array}$ & 25,1 & 3,3 & 47,2 & 21,5 & 0,8 & $-7,1$ & 2,4 & $-16,6$ & 17,1 \\
\hline $\begin{array}{l}\text { \% que realizam internação de } \\
\text { pessoas com HIV no SUS }\end{array}$ & 86,3 & 7,6 & 89,9 & 15,0 & 47,9 & $-5,2$ & 46,3 & $-14,7$ & 63,1 \\
\hline $\begin{array}{l}\text { Média de exames de contagem } \\
\text { de células T-CD4+/CD8+ por } \\
\text { paciente ano }\end{array}$ & 1,7 & - & $1,8 * \star$ & - & 1,7 & - & 1,7 & - & 1,7 \\
\hline $\begin{array}{l}\text { \% que realizam exames de } \\
\text { contagem de células T-CD4/CD8 } \\
\text { por paciente/ano acima da média } \\
\text { nacional }\end{array}$ & 47,5 & $-1,4$ & 66,7 & 7,9 & 42,9 & $-3,0$ & 47,2 & $-4,1$ & 52,0 \\
\hline \multicolumn{10}{|l|}{ Programas de AIDS } \\
\hline $\begin{array}{l}\text { \% incluídos na Política de } \\
\text { Incentivo }\end{array}$ & 43,4 & 7,0 & 66,5 & 26,5 & 0,4 & $-9,2$ & 2,0 & $-22,0$ & 24,3 \\
\hline
\end{tabular}

CTA: Centros de Testagem e Aconselhamento.

* Diferença significativa entre os dois grupos;

** Grupo com média significativamente maior que os outros grupos. 
permitindo identificar e caracterizar espaços geográficos prioritários para a intervenção, e evidenciar desigualdades existentes no acesso à saúde e nas formas de adoecer e morrer. Sua utilização na produção do conhecimento em AIDS, contudo, permanece limitado, dificultando, tanto a compreensão de como formas específicas de organização social e do sistema de saúde produzem distintos perfis de ocorrência da doença no país quanto o reconhecimento das possibilidades e restrições do uso em estudos epidemiológicos dos sistemas de informações gerados a partir das ações de assistência e prevenção.

No que tange especificamente a este estudo, há de se considerar que sua abrangência pode ter sido limitada pela: (1) inexistência de fontes secundárias que compreendem o conjunto das ações públicas e da sociedade civil desenvolvidas no contexto municipal, como projetos de intervenção para populações específicas e a cobertura da rede de saúde suplementar para a oferta do diagnóstico e o tratamento de pessoas com HIV; (2) a impossibilidade de caracterizar a multiplicidade de populações e situações de risco presentes em dado território; (3) a eventual irregularidade na notificação de casos, devido à subnotificação ou à migração de casos ocorridos em municípios de pequeno e médio portes para cidades que concentram a rede de atenção; e (4) o fato de os casos de AIDS representarem infecções ocorridas, em média, há 7 ou 10 anos.

Em contrapartida, os achados apresentados demonstraram uma significativa consistência, observando relações plausíveis entre os indicadores analisados e a ocorrência de diferentes perfis epidemiológicos no país. Nesse aspecto, estudos anteriores já haviam demonstrado a ocorrência de diversas e de diferentes epidemias de AIDS no Brasil 4,6,8, relacionando-as às heterogeneidades regionais e estruturais existentes no país ${ }^{13}$. Os achados do presente estudo acrescentaram a esse mapa as desigualdades existentes na implantação da resposta ao HIV e na presença de situações que podem favorecer magnitudes e tendências específicas de ocorrência da AIDS, permitindo a identificação de um conjunto de municípios que devem ser considerados prioritários para as políticas de saúde.

Sobressaem, nesse contexto, os municípios que apresentaram epidemias de grande magnitude e taxas anuais de incidência em crescimento, sustentadas por um binômio que conjugou maior prevalência de situações de risco e uma relativa baixa capacidade de resposta à epidemia de HIV. Ressalta-se que esses municípios foram os únicos a apresentar crescimento em todas as categorias de transmissão e, mantida a ten- dência, em médio prazo poderão representar as maiores taxas de incidência de AIDS do país.

Em outro pólo, observaram-se as cidades com epidemias de grande magnitude e tendência de estabilização/redução, que ocorreu apesar das mesmas terem sido caracterizadas pela significativa concentração de situações que favoreceram o risco de infecção. Nesse grupo, os resultados sugerem, assim, que o principal diferencial para a redução/estabilização da epidemia foi o grau de implantação da resposta à epidemia, ocorrida nesses municípios com maior intensidade na área de atenção às pessoas com HIV.

As desigualdades foram ainda mais ressaltadas quando analisada a distribuição espacial dos diferentes perfis epidemiológicos identificados, onde, de um lado, se observou as regiões Norte e Nordeste e os municípios de médio porte com epidemias em crescimento e uma relativa baixa capacidade de resposta. E, de outro, as regiões Sudeste, Sul e os maiores centros urbanos do país, caracterizados por possuírem epidemias sob maior controle.

Esses achados nos remetem, fundamentalmente, a três ordens de questões. A primeira é que as desigualdades observadas devido à concentração da resposta a AIDS reproduzem e podem estar sendo reproduzidas, em certa medida, pela própria organização do SUS, que também concentra uma maior capacidade de gestão e de serviços, especialmente de média e alta complexidades, nas regiões Sul e Sudeste e em municípios de maior porte populacional ${ }^{14}$. Nesses locais também se observa a utilização mais intensa dos serviços de atenção e uma melhor auto-avaliação do estado de saúde da população 15 .

Adicionalmente, o modelo de organização do sistema de saúde, com privilégio da atenção individual, pode contribuir para a menor participação das ações de promoção e prevenção na resposta ao HIV, quando comparado ao volume das ações realizadas na área assistencial. Nesse sentido, ressalta-se que os poucos estudos que analisaram a extensão dessas ações 5,16 no Brasil já haviam destacado as limitações existentes, especialmente quando executadas no âmbito das secretarias de saúde, devido, em parte, ao reduzido número de profissionais, à descontinuidade das ações e à desconexão entre realidade epidemiológica e os programas desenvolvidos. Mesmo as ações em escolas, uma das prioridades da resposta ao HIV no Brasil, possuem características que reduzem o seu impacto, por não garantirem amplo acesso aos preservativos e priorizarem ações pontuais e dirigidas somente para o aumento da informação ${ }^{5}$.

Há de se destacar, também, que a maior freqüência das ações de DST e AIDS na atenção bá- 
sica observada em municípios com epidemias de pequena magnitude pode ser compreendida a partir das especificidades deste nível de atenção no país. Atualmente, mesmo com os significativos investimentos realizados para alterar o modelo de atenção predominante 17 , a cobertura da atenção básica permanece relativamente baixa em regiões como as Sul e Sudeste e em municípios com maior número de habitantes, enquanto apresenta indicadores mais favoráveis no Norte, Nordeste e em cidades de médio e pequeno portes 18 . Esse aspecto pode, assim, restringir a importância e o alcance desse nível de atenção nas ações preventivas e assistenciais dirigidas para o controle da AIDS no país.

A segunda ordem de questões a ser abordada refere-se à relação existente entre redução/ estabilização das taxas de incidência e o maior grau de implantação da resposta, ocorrida especialmente nos municípios com epidemias de grande magnitude. Poder-se-ia considerar a hipótese de que a redução/estabilização estaria relacionada às epidemias mais antigas, localizadas nas regiões Sul e Sudeste 4,6 e ao esgotamento da transmissão do HIV em grupos específicos, como os usuários de drogas injetáveis 19. Contudo, é importante sublinhar que essas localidades foram as primeiras a organizar a resposta a AIDS no país 20 , tendo sido ampliadas e fortalecidas a partir de meados dos anos de 1990 com recursos oriundos de empréstimo no Banco Mundial 21 e dos repasses do Ministério da Saúde para o fortalecimento de ações descentralizadas 22. Esses recursos foram investidos, entre outras atividades, na criação da rede de atenção e projetos de prevenção.

Nesse sentido, a disponibilidade de serviços assistenciais pode ter tido um papel estratégico na redução de novos casos. Estudos recentes têm demonstrado que o diagnóstico da infecção, que é ofertado nessas regiões com maior intensidade, e o início do tratamento por ARV de forma oportuna contribuem para a redução da transmissão do HIV, devido à redução da carga viral e à maior probabilidade de pessoas em seguimento clínico adotarem práticas seguras para a prevenção 23,24 .

Entretanto, a excessiva concentração da rede assistencial pode contribuir para a piora da qualidade dos serviços. Por exemplo, o número médio de exames de contagem de célula T-CD4+/CD8+ por paciente observada neste estudo é, aproximadamente, $50 \%$ inferior ao preconizado pelo Ministério da Saúde e a proporção de pacientes com início de tratamento tardio permanece elevada, superando o índice de $40 \% 7$.

A terceira ordem de questões refere-se ao perfil epidemiológico analisado. No Brasil, tem adquirido importância a conceituação proposta pelo United Nations Joint Programme on HIV/ AIDS (UNAIDS) de que em determinados países, como o nosso, a epidemia se circunscreve a grupos específicos, apresentando baixas taxas de prevalência na população em geral 4,25 . Assim, a concentração das situações que podem facilitar o risco de infecção nos maiores centros urbanos, identificada neste estudo, poderia explicar a ocorrência da maior parte dos casos em um número relativamente reduzido de municípios do país, restringindo a possibilidade de municípios de pequeno porte apresentarem epidemias de grande magnitude, excetuando situações específicas, como, por exemplo, a localização dos mesmos em rotas de transporte de mercadorias ou tráfico de drogas.

Ganha dimensão para a resposta brasileira, nesse contexto, as altas proporções de municípios que apresentaram situações relacionadas ao uso de drogas, às desigualdades entre gêneros e à existência de unidades prisionais, que estão relacionadas ao aumento da magnitude da epidemia em dado território. No que tange ao uso de drogas, ressalta-se a crescente importância do uso de crack para transmissão do HIV, estando associado à redução do uso do preservativo, ao aumento da prática comercial do sexo e à mortalidade por AIDS 26. Essas situações podem colaborar para aumentar nessas cidades a transmissão sexual do HIV entre homens e mulheres.

Merece também destaque as taxas de crescimento do número de novos casos entre mulheres, que foram as mais altas em todos os perfis epidemiológicos observados e corroboraram estudos que analisaram a tendência da epidemia de AIDS no país 4 . Os achados reforçam a hipótese de que essa circunstância pode estar relacionada às diferenças entre gêneros 27 , que, como observado, são mais expressivas nos locais com grandes epidemias.

O conjunto dos achados deste estudo evidencia, nessa circunstância, que para a ampliação dos resultados obtidos na resposta brasileira ao HIV será necessário estender as ações programáticas a municípios estratégicos, como aqueles que apresentam ocorrências de maior magnitude e tendência de crescimento. Assim como, deverá aprimorar o modelo de intervenção, conferindo maior peso às ações de prevenção, sem que haja prejuízo às atividades de atenção à saúde de pessoas com HIV. Caso isso não ocorra, o acesso às tecnologias disponíveis e aos benefícios advindos da resposta brasileira continuará ocorrendo especialmente nas áreas mais desenvolvidas e com melhor organização do sistema de saúde. 


\section{Resumo}

Identificar diferentes perfis da epidemia de AIDS no Brasil relacionando-os à organização do setor saúde, às situações relacionadas ao risco de infecção e ao grau de implantação da resposta. Municípios brasileiros foram agrupados segundo magnitude e tendência da epidemia entre 2002 e 2006, e estudados por meio de indicadores obtidos em bases de dados secundários. Municípios com epidemias de grande magnitude (39\%) apresentaram um maior número de situações associadas ao risco e os com tendência de aumento da incidência (11,5\%) menor grau de implantação da resposta. Cidades com grandes epidemias que reduzem/ estabilizam concentraram 68,6\% dos Centros de Testagens, 75,8\% dos ambulatórios e 81,4\% dos exames anti-HIV feitos no sistema de saúde. Ações preventivas em escolas e na atenção básica apresentaram baixa cobertura comparativamente à área assistencial. Diferenças entre regiões foram observadas. Desigualdades no grau de implantação da resposta ao HIV podem contribuir para a ocorrência de diferentes perfis da epidemia no país.

Síndrome da Imunodeficiência Adquirida; Desigualdades em Saúde; Desenvolvimento de Programas; Risco

\section{Colaboradores}

A. Grangeiro e M. M. L. Escuder participaram da concepção original, análise dos dados e redação do artigo. E. A. Castilho participou da análise dos dados e da redação do artigo.

\section{Agradecimentos}

À equipe das Unidades de Planejamento, Departamento Nacional de DST, AIDS e Hepatites Virais e do Programa de AIDS de São Paulo, especialmente, Sérgio D’Ávila, Maria Alice Tironi, Sandra Regina Miguel, Sara Romera da Silva e Vilma Cervantes, pelas contribuições nas discussões e pelo fornecimento de informações e dados; a Paulo Roberto Teixeira pela análise crítica; e a Renata Levy, pela colaboração na análise dos dados.

\section{Referências}

1. Silva JB, Barros MBA. Epidemiologia e desigualdade: notas sobre a teoria e a história. Rev Panam Salud Pública 2002; 12:375-83.

2. Ayres JRCM, França Junior I, Calazans GJ, Saletti Filho HC. O conceito de vulnerabilidade e as práticas de saúde: novas perspectivas e desafios. In: Czeresnia D, Freitas CM, organizadores. Promoção de saúde: conceitos, reflexões, tendências. $3 \underline{a} \mathrm{Ed}$. Rio de Janeiro: Editora Fiocruz; 2005. p. 117-40.

3. Mann JM, Tarantola DJM, Netter TW. AIDS in the World. Cambridge: Harvard University Press; 1992.

4. Fonseca MG, Bastos FI. Twenty-five years of the AIDS epidemic in Brazil: principal epidemiological findings, 1980-2005. Cad Saúde Pública 2007; 23 Suppl 3:S333-44.
5. Paiva V, Pupo LR, Barboza R. O direito à prevenção e os desafios da redução da vulnerabilidade ao HIV no Brasil. Rev Saúde Pública 2006; 40 Suppl:109-19.

6. Szwarcwald CL, Bastos FI, Esteves MAP, Andrade CLT. A disseminação da epidemia da AIDS no Brasil, no período de 1987-1996: uma análise espacial. Cad Saúde Pública 2000; 16 Suppl 1:7-19.

7. Souza Jr. PRB, Szwarcwald CL, Castilho EA. Delay in introducing antiretroviral therapy in patients infected by HIV in Brazil, 2003-2006. Clinics 2007; 62:579-84.

8. Dourado I, Veras MASM, Barreira D, Brito AM. Tendências da epidemia de AIDS no Brasil após a terapia anti-retroviral. Rev Saúde Pública 2006; 40 Suppl:9-17. 
9. França Junior I, Calazans G, Zucchi EM. Grupo de estudos em população, sexualidade e AIDS. Mudanças no âmbito da testagem anti-HIV no Brasil entre 1998 e 2005. Rev Saúde Pública 2008; 42 Suppl 1:84-97.

10. Vieira S. Introdução à bioestatística. Rio de Janeiro: Editora Campus; 1980.

11. Grangeiro A, Escuder MML, Castilho EA. Magnitude e tendência da epidemia da AIDS em municípios brasileiros de 2002-2006. Rev Saúde Pública 2010; 44:430-41.

12. Pereira JCR. Análise de dados qualitativos: estratégias metodológicas para as ciências da saúde, humanas e sociais. 3a Ed. São Paulo: Edusp; 2001.

13. Parker R, Camargo Jr. KR. Pobreza e HIV/AIDS: aspectos antropológicos e sociológicos. Cad Saúde Pública 2000; 16 Supp1:S89-102.

14. Siqueira SAV, Senna MCM, Oliveira PTR, Pinto LF. Descentralização e assistência à saúde no Brasil: a oferta de serviços nos anos 90. Saúde Debate 2002; 26:25-36.

15. Lima JC, Azoury EB, Bastos LHCV, Coutinho MM, Pereira NM, Ferreira SCC. Desigualdades no acesso e utilização de serviços de saúde no Brasil. Saúde Debate 2002; 26:25-36.

16. Cáceres CF. Intervenciones para la prevención del VIH y ITS en América Latina y Caribe: una revisión de la experiencia regional. Cad Saúde Pública 2004; 20:1468-85.

17. Malamed C, Costa NR. Inovações no financiamento federal à Atenção Básica. Ciênc Saúde Coletiva 2003; 8:393-401.

18. Facchini LA, Piccini RX, Tomasi E, Thumé E, Silveira DS, Siqueira FV, et al. Desempenho do PSF no Sul e no Nordeste do Brasil: avaliação institucional e epidemiológica da Atenção Básica à Saúde. Ciênc Saúde Coletiva 2006; 11:669-81.
19. Bastos FI, Bongertz V, Teixeira SL, Morgado MG, Hacker MA. Is human immunodeficiency virus/ acquired immunodeficiency syndrome decreasing among Brazilian injection drug users? Recent findings and how to interpret them. Mem Inst Oswaldo Cruz 2005; 100:91-6.

20. Grangeiro A, Laurindo-Silva L, Teixeira PR. Resposta à AIDS no Brasil: contribuições dos movimentos sociais e da reforma sanitária. Rev Panam Salud Pública 2009; 26:87-94.

21. Berkman A, Garcia J, Muñoz-Laboy M, Paiva V, Parker R. A critical analysis of the Brasilian response to HIV/AIDS: lessons learned for controlling and mitigating the epidemic in developing countries. Am J Public Health 2005; 95:1162-72.

22. Taglieta MFB. Avaliação da implantação do incentivo no âmbito do Programa Nacional de HIV/AIDS e outras DST em dois municípios do Estado de São Paulo [Dissertação de Mestrado]. Rio de Janeiro: Escola Nacional de Saúde Pública, Fundação Oswaldo Cruz; 2006.

23. Valdiserri RO, Holtgrave DR, West GR. Promoting early HIV diagnosis and entry into care. AIDS 1999; 13:2317-30.

24. Fisher M. Late diagnosis of HIV infection: major consequences and missed opportunities. Curr Opin Infect Dis 2008; 21:1-3.

25. Barbosa Junior A, Szwarcwald CL, Pascon ARP, Souza Junior PB. Tendências da epidemia de AIDS entre subgrupos sob maior risco no Brasil, 19802004. Cad Saúde Pública 2009; 25:727-37.

26. Duailibi LB, Ribeiro M, Laranjeira R. Profile cocaine and crack users in Brazil. Cad Saúde Pública 2008; 24 Suppl 4:S545-57.

27. Dowsett GW. Algumas considerações sobre sexualidade e gênero no contexto da AIDS. Questões de Saúde Reprodutiva 2006; 1:39-49.

Recebido em 10/Dez/2009

Versão final reapresentada em 10/Mai/2010

Aprovado em 20/Mai/2010 